\title{
Connectivity vs Capacity in Dense Ad Hoc Networks
}

\author{
Olivier Dousse \\ School of Computer and \\ Communication Sciences \\ EPFL \\ Lausanne, Switzerland \\ Email: olivier.dousse@epfl.ch
}

\author{
Patrick Thiran \\ School of Computer and \\ Communication Sciences \\ EPFL \\ Lausanne, Switzerland \\ Email: patrick.thiran@epfl.ch
}

\begin{abstract}
We study the connectivity and capacity of finite area ad hoc wireless networks, with an increasing number of nodes (dense networks). We find that the properties of the network strongly depend on the shape of the attenuation function. For power law attenuation functions, connectivity scales, and the available rate per node is known to decrease like $1 / \sqrt{n}$. On the contrary, if the attenuation function does not have a singularity at the origin and is uniformly bounded, we obtain bounds on the percolation domain for large node densities, which show that either the network becomes disconnected, or the available rate per node decreases like $1 / n$.
\end{abstract}

\section{INTRODUCTION}

Properties of wireless ad hoc and sensor networks as fundamental as their connectivity and capacity are so challenging, that they require (and may thus strongly depend on) assumptions on the physical features of the radio channels, on the power assignments, on the node locations, on the traffic matrix, to name a few.

The scalability of these properties is of primary concern, hence the results obtained are often of asymptotic nature, valid when the number of nodes $n$ is large enough. This can be achieved in two different ways: either the network is deployed on a finite area $s$, with a sufficiently large node density $\lambda$ (we will speak in this paper of a dense network), or the node density $\lambda$ is kept constant, but the surface $s$ is made sufficiently large (we will then speak of an extended network).

In this paper, we assume that the nodes are located according to a Poisson point process over the plane. We adopt the physical model based on the signal to interference ratio of Gupta and Kumar [1]. All stations are assumed to have the same power, and some attenuation function $l(\cdot)$ is given. Station $A$ can receive a signal from station $B$ if the ratio of the power it receives from $B$ to the total power received from all other stations is above a threshold, denoted by $\beta$.

By analogy with CDMA networks, we introduce some orthogonality factor $\gamma$, which can vary from 0 to 1 , and which stems from the imperfect orthogonality of the codes used in CDMA. The value $\gamma=0$ means that interferences

The work presented in this paper was supported (in part) by the National Competence Center in Research on Mobile Information and Communication Systems (NCCR-MICS), a center supported by the Swiss National Science Foundation under grant number 5005-67322. are neglected or that the codes are perfectly orthogonal, the value $\gamma=1$ corresponds to the other extreme of a narrowband system, where all interfering communications are considered as noise.

A first study of connectivity of the resulting graph, called Signal To Interference Ratio Graph (STIRG), has been made in [2], for extended networks. The STIRG displays essential differences from the graph obtained in the Boolean Model, where $\gamma=0$ : the node degree is bounded instead of being Poisson, and the existence of an edge between two nodes depends not only on the location of these two nodes, but on the location of all others. The main result of that paper was that under attenuation functions with finite support, percolation occurs. That is, the graph contains an infinite component, provided the node density $\lambda$ is large enough, and the orthogonality factor $\gamma$ is small enough. One can thus say that connectivity of ad hoc networks scales well with the size of the network, even in the case of models that take interferences into account.

In this paper, we first complete the study of the curve $\gamma^{*}(\lambda)$ in the 2-dim. parameter space $(\lambda, \gamma)$, which marks the phase transition between the sub-critical phase, where the STIRG contains almost surely only finite-sized, disconnected clusters, and the super-critical phase, where the STIRG contains an infinite cluster with non-zero probability.

Contrary to [2], we do not assume that the attenuation function is restricted to have a finite support, and we consider the asymptotic connectivity of $\lambda \rightarrow \infty$, hence this paper focuses on dense networks.

We show that the shape of the attenuation function $l(\cdot)$ has a crucial impact on this curve. In particular, we show that the most popular attenuation function, namely the power law function $l(x)=x^{-\alpha}$, where $\alpha$ is the path loss exponent (which must be larger than 2 [2]) enjoys particular scaling properties and a singularity at the origin that lead to a monotonically increasing curve $\gamma^{*}(\lambda)$.

However, in the most realistic case where $l(x)$ is bounded from above, the curve $\gamma^{*}(\lambda)$ is non monotonic. Using site percolation [3], we prove that for $\lambda \rightarrow \infty, \gamma^{*}(\lambda)$ decreases at least as fast as $1 / \lambda$. Figure 1 shows the difference between the two curves for the two attenuation functions $l(x)=x^{-3}$ and $l(x)=\min \left(1, x^{-3}\right)$, respectively. This means that the 
absence of singularity of the attenuation function at the origin is an essential factor affecting connectivity in dense networks.

The vast majority of experimental power-law attenuation models are valid at large scales, relatively far away from the emitting antenna. Experimental models valid at a smaller scale (from a few meters to a few kilometers) are usually piecewise power laws, with different path loss exponents, increasing with the distance from the antenna [4]. For example, [5] found by regression a piecewise power law with two segments, the first one having a path loss exponent between 1.4 and 2 for a range of distances between 1 and 500 meters, and the second one having a path loss exponent between 4 and 10 for a range of distances between 500 and 1000 meters. The attenuation function decreases slower close to the origin than a strict power law, and is bounded from above for physical reasons. For models neglecting interferences, and for extended networks, these changes from a strict power law attenuation function may be only second order effects on the performance of the network. For models of dense networks taking interferences into account, such as the STIRG, this paper will show that it is no longer so.

Instead of deleting edges in the STIRG when the Signal to Noise ratio is too low, which may result in a very poor connectivity as the STIRG will be in a sub-critical phase if the threshold $\beta$ is set to high, an alternative is to decrease the transmission rate. We will see that if the attenuation function is bounded, the threshold $\beta$ must decrease like $1 / \lambda$ to maintain connectivity. There is therefore a trade-off between capacity and connectivity. By using a TDMA scheme allowing only a fraction of $1 / t$ nodes to emit simultaneously, one decreases the amount of interferences, but also the rate available per node. We prove that such a simple scheme does increase the connectivity, at the expense of decreased capacity, which illustrates again the trade-off between capacity and connectivity. Finally, we prove that the total transport capacity does not increase with $n$ for bounded attenuation functions, whose support is larger than the entire domain of size $s$, for any scheduling strategy.

The paper is structured as follows. After having reviewed some of the results on connectivity and capacity in the literature in the next section, we formally define the STIRG model in Section III and the various assumptions made on the model. We then review its scaling properties in Section IV, which will be useful to examine the connectivity of dense networks with a unbounded, power law attenuation function in Section V. We next move to the central section of the paper, Section VI, devoted to the asymptotic connectivity of the network and a bound on capacity when the attenuation function is bounded from above. Finally, we draw some conclusions and future perspective in Section VII.

\section{RELATED WORK}

Connectivity and capacity are fundamental properties of ad hoc networks.

Connectivity has received quite a lot of attention in the previous decade already, in the context of packet radio networks,

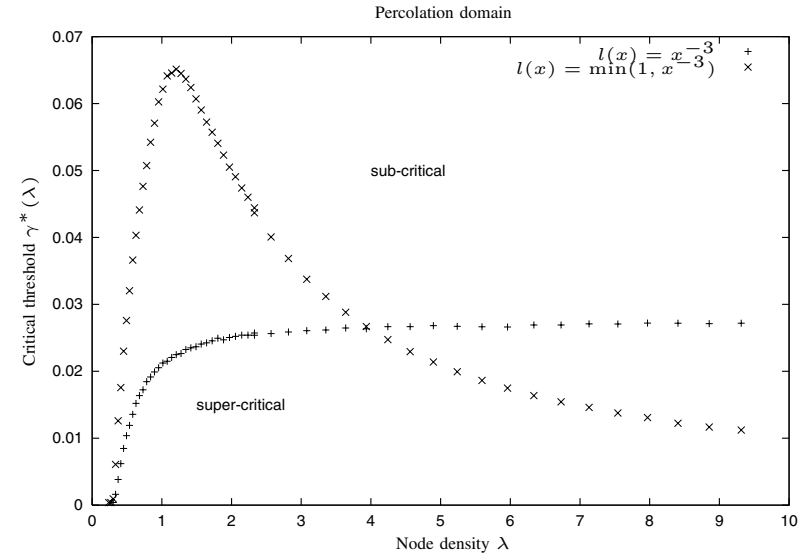

Fig. 1. Percolation domain computed by simulation, with $l(x)=x^{-3}$ and $l(x)=\min \left(1, x^{-3}\right)$. The parameters are $P=100,000, \beta=1$, and $N_{0}=10,000$

and has gained renewed interest recently in the context of ad-hoc and sensor networks. Many results apply to the full connectivity of a network made of a finite number of nodes. However, there are numerous papers on the connectivity of infinite networks.

In extended networks, the connectivity problem is related to percolation theory, which is to find the probability that a node belongs to an infinite cluster of nodes. The most popular model is the Poisson Boolean model, where node locations follow a Poisson distribution, as in our work, but where only the distance between two nodes determines the existence of a link between them. This model is a particular case of the STIRG for $\gamma=0$, and was introduced by Gilbert [6], who started the field of continuum percolation. Despite the apparent simplicity of the Poisson Boolean model, the exact value of the critical density $\lambda^{*}$ at which the transition occurs is still an open problem. Some bounds on $\lambda^{*}$ have been obtained analytically in [6], [7], [8], and numerically by many others [9].

Percolation of a clustered wireless network, in which the users (clients), who are distributed according to a Poisson process, are all covered by base stations that can connect to each other by a wireless link, is studied in [10]. This model boils down to the Poisson Boolean Model if one base station is placed at each client. The opposite case where base stations are connected to each other by a wired link, but do not ensure a complete coverage of the users, is studied in [11].

The assumption that two nodes are connected to each other if and only if their distance is less than or equal to some predefined radius is certainly too simplistic. In reality, propagation in a real environment yields much more complex and irregular coverage shapes than circles. Interestingly, it appears that this irregularity makes the network percolate at lower values of the density than the Boolean model [12]. The later appears thus as a conservative model for noisy channels. When interferences are taken into account however, this is no longer true: for the same patterns, the Boolean model 
may have an infinite component of the connectivity graph, whereas the STIRG could have no infinite component, or even no connectivity at all [2]. This physical model was analyzed in [13] in the infinite plane case under Poisson assumptions within the context of CDMA networks. The level sets defining the contours around a node where the Signal to Interference has the same value can indeed have a very convoluted shape.

In dense networks, the full connectivity of the graph has been shown to hold with probability 1 when the distance $r$ below which nodes can connect decreases at a rate slower than $\sqrt{\log n / n}$, with the number of nodes $n$ tending to $\infty$ in [14].

A similar problem, where the power of a node is adjusted so that it can have a required number of neighbors, is studied in [15]. When the node locations are restricted to be at the vertices of a grid, instead of being scattered on the entire area as in the previous examples, results on the asymptotic connectivity of the network have been obtained in [16].

The capacity of wireless ad hoc networks belongs to the very difficult field of multi-users information theory. In their seminal paper, Gupta and Kumar [1] proved that the aggregate transport capacity, defined as the sum of all the link capacities multiplied by the distances between the source and destination, is of the order of $\sqrt{n}$ bit-meter per second, for dense networks, point-to-point codes, a uniform traffic matrix and a power law attenuation with a path loss exponent $\alpha>2$. This means that the rate per user decreases as $1 / \sqrt{n}$ and eventually reaches 0 for $n \rightarrow \infty$. These results rely on the assumption that the signal to noise and interference ratio exceeds some threshold $\beta$, as in our paper. This assumption, which may make sense in practice but is not truly of an information theoretic nature, is not needed, and Xie and Kumar [17] have proven that for attenuation functions of the type $e^{-a x} / x^{\alpha}$, with either $a>0$ or $\alpha>3$, the aggregate transport capacity is of the order of $n / \sqrt{\log n}$ bit-meter per second, under the assumption that the minimum distance between any pair of nodes is bounded below by some constant. This restriction forces the domain size $s$ to grow with $n$, hence that capacity result applies to extended networks. These results were extended to power law attenuation functions with $\alpha>2$ in [18], for extended networks without the minimum distance restriction. They encompass therefore the case where nodes are distributed as a Poisson process.

These general results are more optimistic if one considers some particular scenarios. For example, if there is only one active source/destination pair, while the $n-2$ other nodes act as possible relays, the previous results would predict a constant capacity, independent of $n$, whereas allowing arbitrarily complex coding, this capacity is of the order of $\log n$ [19]. Another example is the increased capacity obtained when the nodes are mobile: with one hop relaying, the total transport capacity can be of the order of $n$ [20]. Finally, the benefit of hybrid multi-hop cellular networks is investigated for the so-called protocol model in [21].

To summarize, one can notice that most of the papers study the connectivity and capacity of ad hoc networks mainly for the Boolean and physical models with power law attenuation functions. We show in this paper that these properties are dramatically changed when the attenuation function is bounded at the origin.

\section{MODEL}

On a given compact subset $S$ of $\mathbb{R}^{2}$ of area $s$, we consider a two-dimensional Poisson process $\Phi_{\lambda}$ of intensity $\lambda$. The points of the process are the nodes of the network. For any subset $E \subset S$, we will denote by $\Phi_{\lambda}(E)$ the number of points located in $E$. This number is a Poisson random variable of parameter $\lambda e$, where $e$ is the area of $E$. In particular, the average total number of points in $S$ is

$$
n:=\lambda s .
$$

To describe the available links between these nodes, we use the physical model as described in [1]. We define the signal to noise and interference ratio (SINR) of the signal received by Node $j$ from Node $i$ as

$$
\beta_{i j}=\frac{P_{i} l\left(\left\|x_{i}-x_{j}\right\|\right)}{N_{0}+\gamma \sum_{k \neq i, j} P_{k} l\left(\left\|x_{k}-x_{j}\right\|\right)},
$$

where $P_{k}$ is the power emitted by Node $k, l(\cdot)$ is the attenuation function of the signals, $N_{0}$ is the ambient noise, and $\gamma$ is the orthogonality factor between codes.

To study the connectivity of the network, we make the assumption the two nodes are able to communicate directly if the SINR of the signal they receive from each other is above a certain threshold $\beta$ :

Definition 1: Node $i$ is directly connected to Node $j$ if

$$
\beta_{i j}>\beta \text { and } \beta_{j i}>\beta
$$

With this definition, we obtain a non-oriented graph that describe the connectivity of the network. We call this graph STIRG (Signal to interference ratio graph), as proposed in [2], and denote it by $\mathcal{G}(\lambda, \gamma)$.

Throughout the paper, we make several assumptions on the attenuation function $l(\cdot)$. The first assumption is that $l(x)$ has to decrease faster than $x^{-2}$ when $x$ tends to infinity. It was proven in [2] that the STIRG model does not make sense for large networks otherwise. In Section V, we assume that $l(x)=x^{-\alpha}$, for some $\alpha>2$. In Section VI, we assume that $l(x)$ is decreasing, and that $l(0)=M<\infty$.

Moreover, for the sake of simplicity, while studying connectivity, we will make the assumption that all the nodes emit with a common power $P$. This assumption does actually not essentially change the behavior of the model, compared to the case where each node $i$ emit with a particular power $P_{i} \in\left[P_{\min }, P_{\max }\right]$, with $P_{\min }>0$ and $P_{\max }<\infty$. The assumption that the minimal emitting power $P_{\min }$ is strictly positive will be relaxed in Sections VI-B and VI-C.

For the transport capacity, we do not require any minimal SINR, but we assume that the available rate on a link is given by the Gaussian channel model (the bandwidth has been normalized to one for the sake of simplicity)

$$
C\left(\beta_{i j}\right)=\frac{1}{2} \log \left(1+\beta_{i j}\right) \leq \frac{1}{2} \beta_{i j} .
$$


We consider the model where communications take exclusively place in a peer-to-peer manner. This means that at each instant, each node emit data towards at most one other node, and that the latter is the only node listening to the former. To compute an upper bound to the actual transport capacity of the network, we will thus sum the transport capacity provided by each node. The transport capacity of an individual node $i$, assuming that it transmits data towards Node $j$, is defined as the capacity of the link $C\left(\beta_{i j}\right)$ times the distance between source and destination $\left\|x_{i}-x_{j}\right\|$. We will denote hereafter by $r(k)$ the destination of the data transmitted by Node $k$. In this example, we have thus $r(i)=j$.

\section{SCALING PROPERTIES}

In this section, we study the symmetry properties of the STIRG model with respect to scaling. We derive a bunch of useful tools that we will need later to study its properties.

\section{A. The Poisson point process}

We start by defining the dilation operator $h_{a}$ of $\mathbb{R}^{2}$, with $a \in \mathbb{R}_{+}$as follows:

$$
\begin{aligned}
h_{a}: \mathbb{R}^{2} & \longrightarrow \mathbb{R}^{2} \\
x & \longmapsto a x
\end{aligned}
$$

Let us also denote the probability space by $\Omega$. If we apply $h_{a}$ to the Poisson point process, we obtain another Poisson point process, but with density $\lambda / a^{2}$ :

$$
h_{a} \Phi_{\lambda}=\Phi_{\lambda / a^{2}} .
$$

In particular, one can define $\Phi_{\lambda}$ and $\Phi_{\lambda / a^{2}}$ such that if we apply $h_{a}$ to a particular realization of $\Phi_{\lambda}$, we obtain the corresponding realization of $\Phi_{\lambda / a^{2}}$ :

$$
h_{a} \Phi_{\lambda, \omega}=\Phi_{\lambda / a^{2}, \omega} \quad \forall \omega \in \Omega .
$$

Thus if $x_{1}$ and $x_{2}$ are two points of $\Phi_{\lambda, \omega}, a x_{1}$ and $a x_{2}$ belong to $\Phi_{\lambda / a^{2}, \omega}$. In particular, the distance between these points is multiplied by $a$ when we apply $h_{a}$.

This technique, called coupling, allows us to compare Poisson processes of different density. Briefly said, decreasing the node density by a factor $a^{2}$ is equivalent to multiply all the distances by $a$ (see [7] pp 28-31 for more details on scaling and coupling).

\section{B. The physical model}

If we introduce now on top of the Poisson process our physical model, we can derive its scaling properties. Let us construct the STIRG for a given node density $\lambda$ and attenuation function $l$, and pick two nodes $x_{1}, x_{2}$ that are directly connected. In other words, (2) holds for $i=1$ and $j=2$. We apply then $h_{a}$ to the entire graph. We want to show that the dilated (respectively contracted, if $a<1$ ) graph is another STIRG. We want thus to derive a condition on $a x_{1}$ and $a x_{2}$ of the same form as (2). Let us try with a new attenuation function $l^{\prime}(\cdot)$. We want thus

$$
\frac{P_{1} l^{\prime}\left(\left\|a x_{1}-a x_{2}\right\|\right)}{N_{0}+\gamma \sum_{k \neq 1,2} P_{k} l^{\prime}\left(\left\|a x_{k}-a x_{2}\right\|\right)}>\beta \text {. }
$$

This condition is always true if we choose $l^{\prime}$ such that

$$
l^{\prime}(a x)=l(x) .
$$

Consequently, the STIRG $\mathcal{G}(\lambda, \gamma)$ with attenuation function $l(x)$ is equivalent by dilation to $\mathcal{G}\left(\lambda / a^{2}, \gamma\right)$ with $l^{\prime}(x)=$ $l(x / a)$.

\section{Symmetric attenuation functions}

We saw that rescaling the STIRG model implies to modify the attenuation function. We now look for a specific category of these functions, such that the relative impact of rescaling on the transmission gains is constant. In other words, if Node 1 sends to Node 2, and Node 3 sends to Node 4, we would like to have

$$
\frac{l\left(\| a x_{1}-a x_{2}||\right)}{l\left(\left\|x_{1}-x_{2}\right\|\right)}=\frac{l\left(\| a x_{3}-a x_{4}||\right)}{l\left(\left\|x_{3}-x_{4}\right\|\right)},
$$

or equivalently

$$
\frac{l(a x)}{l(x)}=\frac{l(a y)}{l(y)}, \quad \forall a, x, y>0 .
$$

We show in appendix that the only class of functions that has this property happens to be the commonly used power law function $l(x)=c x^{-\alpha}$. This implies that the model acquires specific symmetry properties with respect to scaling when this kind of attenuation function is considered. In the scaled graph, we have (see Appendix)

$$
\frac{P_{1} a^{-\alpha}|| x_{1}-x_{2} \|^{-\alpha}}{N_{0}+\gamma \sum_{k \neq 1,2} P_{k} a^{-\alpha}\left\|x_{k}-x_{2}\right\|^{-\alpha}}>\beta,
$$

and thus

$$
\frac{P_{1}\left\|x_{1}-x_{2}\right\|^{-\alpha}}{a^{\alpha} N_{0}+\gamma \sum_{k \neq 1,2} P_{k}\left\|x_{k}-x_{2}\right\|^{-\alpha}}>\beta .
$$

Rescaling the network in this case is therefore equivalent to multiply the background noise by a factor $a^{\alpha}$. The attenuation function remains unchanged.

\section{Full connectivity and percolation}

Let us conclude this section by a note on the difference between full connectivity and connectivity in the super-critical phase. For a given network topology, it is always possible to find parameters to achieve full connectivity of the network. In this paper, the position of the nodes is random. Therefore, we want here to predict connectivity as a function of the parameters of the model. Because of the properties of the Poisson point process, it is actually impossible to find parameters such that the probability of fully connectivity is one.

For example, in the Boolean model, it has been shown in [14] that the range of the nodes has to increase roughly like $\sqrt{n}$ to ensure fully connectivity. This means that given a number of nodes $n$, one can choose a range $r(n)$ such that the average fraction of connected nodes is $p(n)$, and

$$
\lim _{n \rightarrow \infty} p(n)=1 \text {. }
$$

However, for finite $n, p(n)$ is always strictly smaller than 1 . 
In this paper, to preserve the ergodic property of the model, we will require that the probability for an arbitrary node to be connected (that is, to belong to the largest connected component of the network) is strictly positive. This requires that the largest connected component contains a non-negligible fraction of the nodes.

It was shown by simulation in [2] (and proven for bounded decreasing attenuation functions over a finite support) that for infinite area networks and for a sufficiently large node density $\lambda$, there exists a critical value $\gamma^{*}(\lambda)$ such that if $\gamma<\gamma^{*}(\lambda)$, the network contains a unique infinite-sized connected component. The network is said to be super-critical in this case (we sometimes also say that percolation occurs). Simulation results show moreover that the fraction of nodes belonging to the infinite component grows rapidly for $\gamma<\gamma^{*}(\lambda)$. On the contrary, if $\gamma>\gamma^{*}(\lambda)$, the network is fragmented into finitesized components, and is said to be sub-critical.

In the case of a finite area network, as in this paper, the picture remains the same. We simply consider a finite sample of the infinite network. The average fraction of nodes in the sample that belong to the infinite cluster is the same as in the infinite network. Therefore, super-criticality implies positive probability of connection. In the sequel, we will thus use percolation to study connectivity.

\section{POWER LAW ATTENUATION FUNCTIONS}

As we saw in Section IV, when $l(x)=x^{-\alpha}$, the model acquires new scaling properties. In particular, increasing the node density by a factor $a^{2}$ is equivalent to multiply the background noise by a factor $a^{-\alpha}$. We show in this section that the critical value $\gamma^{*}(\lambda)$ is an increasing function of the node density $\lambda$ in this particular case, as observed in Figure 1.

Theorem 1: For an attenuation function $l(x)=x^{-\alpha}, \alpha>$ 2 , the critical threshold $\gamma^{*}(\lambda)$ for percolation of the STIRG model, if it exists, is an increasing function of the node density.

Proof: Let $\lambda_{1}$ and $\lambda_{2}$ be two node densities such that $\lambda_{1} \leq \lambda_{2}$. We consider a realization of $\mathcal{G}\left(\lambda_{1}, \gamma\right)$, and pick an edge of the graph, say between Node $i$ and Node $j$. We define also $a=\sqrt{\lambda_{1} / \lambda_{2}} \leq 1$. The SINR at Node $j$ is

$$
\begin{aligned}
\beta_{i j} & =\frac{P l\left(\left\|x_{i}-x_{j}\right\|\right)}{N_{0}+\gamma \sum_{k \neq i, j} P l\left(\left\|x_{k}-x_{j}\right\|\right)} \\
& =\frac{P a^{\alpha} l\left(\left\|x_{i}-x_{j}\right\|\right)}{a^{\alpha} N_{0}+\gamma \sum_{k \neq i, j} P a^{\alpha} l\left(\left\|x_{k}-x_{j}\right\|\right)} \\
& \leq \frac{P l\left(a\left\|x_{i}-x_{j}\right\|\right)}{N_{0}+\gamma \sum_{k \neq i, j} P l\left(a\left\|x_{k}-x_{j}\right\|\right)} .
\end{aligned}
$$

On the other hand, if we apply the contraction $h_{a}$ to the graph, we obtain a node density $a^{2} \lambda_{1}=\lambda_{2}$. The contracted graph is thus the corresponding realization of $\mathcal{G}\left(\lambda_{2}, \gamma\right)$. The condition for the existence of a link between Node $i$ and Node $j$ in $\mathcal{G}\left(\lambda_{2}, \gamma\right)$ is

$$
\beta_{i j}^{\prime}=\frac{P l\left(\left\|x_{i}^{\prime}-x_{j}^{\prime}\right\|\right)}{N_{0}+\gamma \sum_{k \neq i, j} P l\left(\left\|x_{k}^{\prime}-x_{j}^{\prime}\right\|\right)}>\beta,
$$

where the coordinates $x_{k}^{\prime}$ are measured in the contracted space. We have thus for any node $k$

$$
x_{k}^{\prime}=a x_{k} .
$$

Therefore, because of (4),

$$
\beta_{i j}^{\prime}=\frac{P l\left(a\left\|x_{i}-x_{j}\right\|\right)}{N_{0}+\gamma \sum_{k \neq i, j} P l\left(a\left\|x_{k}-x_{j}\right\|\right)}>\beta_{i j}>\beta .
$$

This proves that any link in $\mathcal{G}\left(\lambda_{1}, \gamma\right)$ exists also in $\mathcal{G}\left(\lambda_{2}, \gamma\right)$. Super-criticality of the first implies thus super-criticality of the second. However, we know that $\mathcal{G}\left(\lambda_{1}, \gamma\right)$ is super-critical if $\gamma<\gamma^{*}\left(\lambda_{1}\right)$. Therefore

$$
\gamma<\gamma^{*}\left(\lambda_{1}\right) \Rightarrow \gamma<\gamma^{*}\left(\lambda_{2}\right)
$$

We proved thus that $\gamma^{*}\left(\lambda_{1}\right) \leq \gamma^{*}\left(\lambda_{2}\right)$.

A word of caution is needed at this point, as the existence of a non-trivial curve $\gamma^{*}(\lambda)$ has not been proven to date for power law attenuation functions, and it is only conjectured. However, if it exists, $\gamma^{*}(\lambda)$ enjoys a quite surprising property: even though all nodes are emitting at the same time and thus interfere more and more, increasing the node density helps for connectivity. This result is counter-intuitive, and is due to the very particular attenuation function. In fact, as $l(x)$ tends to infinity when $x$ becomes small, the signal level remains comparable to the interference level when the node density increases.

The particularity of the power law attenuation function also affects the capacity of the network. The case of a finite area network with increasing node density has been studied in [1], with this kind of attenuation function (in the physical model). The result is that the total transport capacity of the network grows at most like $\sqrt{n}$. This is a negative result, but we will see in Section VI-C that the transport capacity remains constant when the node density increases if the attenuation function is bounded.

We conclude that power law attenuation functions lead to scalable connectivity and half-scalable transport capacity in the case of dense ad hoc networks. This is due to their invariance property with respect to dilation.

\section{BOUNDED ATTENUATION FUNCTION}

In this section, we address the case where the attenuation function is uniformly bounded, namely:

$$
l(x) \leq M, \quad \forall x \in \mathbb{R}^{+} .
$$

When the node density becomes high, the average level of interference in the network becomes also high (its average grows linearly with $\lambda$ ). If we assume that the attenuation function is bounded, we see intuitively that communication becomes impossible. One can observe that directly in the expression of the signal-to-noise ratio:

$$
\frac{\overbrace{P l\left(\left\|x_{i}-x_{j}\right\|\right)}^{\text {bounded }}}{N_{0}+\underbrace{\gamma \sum_{k \neq i, j} P l\left(\left\|x_{k}-x_{j}\right\|\right)}_{\text {unbounded }}} .
$$




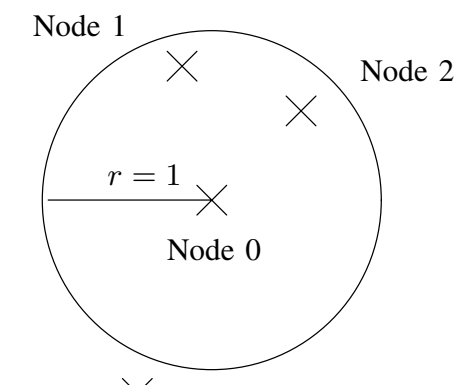

Node $3 X$

Fig. 2. A situation where the truncated attenuation performs better than the unbounded one.

We guess that the critical value $\gamma^{*}(\lambda)$ has to decrease to zero when the node density $\lambda$ increases. Simulation results confirm this intuition, as shown in Figure 1. It is interesting to notice that for moderate densities, the bounded attenuation function leads to a much higher critical threshold than the power law. This is due to the fact that the boundedness at the origin also reduces the interference term.

To see more precisely what happens, let us look at a simple example (depicted in Figure 2), with attenuation function $l(x)=\min \left(1, x^{-\alpha}\right)$ : consider a node (say Node 0 ) and assume that it is connected to its two first neighbors (Nodes 1 and 2). We look at the SINR available from the third neighbor (Node 3 ). When Node 0 listens to Node 3 , most of the interference comes from Nodes 1 and 2. If Node 3 is located at a distance greater than 1 from Node 0 , the strength of its signal at Node 0 is the same as with $l(x)=x^{-\alpha}$. However, if at the same time Nodes 1 and 2 are located at a distance smaller than one (this situation happens with a reasonable probability when $\lambda$ has a suitable value), their signals (taken as interference here) have a magnitude bounded by 1 . Thus, Node 3 has more chance to be connected than in the unbounded attenuation function case.

\section{A. Asymptotic behavior of $\gamma^{*}(\lambda)$}

We will now study the asymptotic behavior of $\gamma^{*}(\lambda)$ when $\lambda$ goes to infinity. We start by finding an upper bound and show that it tends to zero when $\lambda$ increases.

Let us assume that the attenuation function is decreasing:

$$
x_{1} \leq x_{2} \Rightarrow l\left(x_{1}\right) \geq l\left(x_{2}\right)
$$

and

$$
l(0)=M .
$$

Second, we require that $l(x)$ is greater than a positive constant $m$ in the neighborhood of the origin:

$$
l(x) \geq m, \forall x \in[0, \delta],
$$

for some $\delta>0$.

Theorem 2: If the attenuation function is decreasing and such that (5) and (6) hold, there exists a constant $c_{1}<\infty$ such that

$$
\gamma^{*}(\lambda)<\frac{c_{1}}{\lambda} .
$$

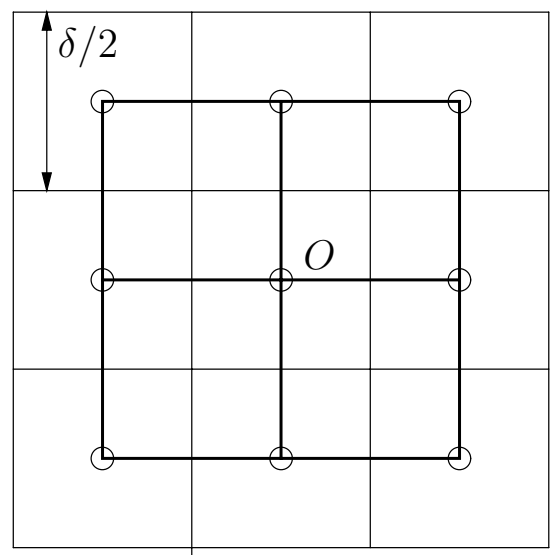

Fig. 3. Mapping of the continuous STIRG model onto a site percolation model

To prove this theorem, we will need the following simple lemma:

Lemma 1: Let $X$ be a Poisson random variable of parameter $\mu$, and $0<\varepsilon<1$ a positive constant. Then

$$
\lim _{\mu \rightarrow \infty} \mathbb{P}[X \leq(1-\varepsilon) \mu]=0 .
$$

Proof: Using Chebycheff inequality:

$$
\mathbb{P}[|X-\mu| \geq \varepsilon \mu] \leq \frac{\operatorname{Var}(X)}{\varepsilon^{2} \mu^{2}}=\frac{1}{\varepsilon^{2} \mu} .
$$

Thus

$$
\lim _{\mu \rightarrow \infty} \mathbb{P}[|X-\mu| \geq \varepsilon \mu]=0,
$$

which implies

$$
\lim _{\mu \rightarrow \infty} \mathbb{P}[X \leq(1-\varepsilon) \mu]=0 .
$$

We then map our network onto a discrete model. We proceed as follows: we divide $\mathbb{R}^{2}$ into squares of edge length $\delta / 2$, as depicted in Figure 3. The centers of these squares form a lattice $\mathbb{Z}^{2}$. We assume furthermore, without loss of generality, that the origin of $\mathbb{R}^{2}$ coincides with the origin of the lattice.

Because of Condition (6), if there are $n$ nodes in a square, the sum of the interferences everywhere in the square is greater than $\mathrm{nm}$. Lemma 2 then follows:

Lemma 2: If there are more than

$$
N^{\prime}=\frac{(1+2 \beta \gamma) M}{\beta \gamma m}
$$

nodes inside a square, all nodes in this square are isolated.

Proof: Pick any node $i$ inside the square, and another node $j$ (inside or outside the square). As $l(\cdot)$ is bounded from above by $M$, we have

$$
P l\left(|| x_{j}-x_{i} \|\right) \leq P M .
$$


Because of (6), we also have

$$
\begin{aligned}
\sum_{k \neq i, j} P l\left(\left\|x_{k}-x_{i}\right\|\right) & \geq \sum_{k \in \text { square }, k \neq i, j} P l\left(\left\|x_{k}-x_{i}\right\|\right) \\
& \geq \sum_{k \in \text { square }} P l\left(\left\|x_{k}-x_{i}\right\|\right)-2 P M \\
& \geq N^{\prime} P m-2 P M \\
& =\beta N^{\prime} N_{0}-2 P M .
\end{aligned}
$$

Therefore we have

$$
\begin{aligned}
\frac{P l\left(\left\|x_{j}-x_{i}\right\|\right)}{N_{0}+\gamma \sum P l\left(\left\|x_{k}-x_{i}\right\|\right)} & \leq \frac{P M}{N_{0}+\gamma\left(\beta N^{\prime} P m-2 P M\right)} \\
& \leq \frac{P M}{\gamma\left(\beta N^{\prime} P m-2 P M\right)} \\
& =\frac{M}{\gamma\left(\beta N^{\prime} m-2 M\right)} .
\end{aligned}
$$

The above expression is clearly smaller than $\beta$ when $N^{\prime}>$ $(1+2 \beta \gamma) M / \beta \gamma m$, which implies that Node $i$ is isolated.

We can now define a site percolation model by declaring a site of $\mathbb{Z}^{2}$ open if the square centered on this point contains at most $2 N^{\prime}$ nodes. It is declared closed otherwise. It is clear that each site is open or closed independently from the others.

We know from percolation theory that if

$$
\mathbb{P}(\text { a site is open })<p_{\text {site }},
$$

the origin belongs to a finite cluster in the discrete model. $p_{\text {site }}$ is the critical site percolation threshold, whose value is around 0.59 (see [3] p.56). The number of nodes inside a square is a Poisson random variable of parameter $\lambda \delta^{2} / 4$. Lemma 1 implies that if for some $0<\varepsilon<1$,

$$
2 N^{\prime} \leq(1-\varepsilon) \lambda \delta^{2} / 4
$$

we have

$$
\lim _{\lambda \rightarrow \infty} \mathbb{P}(\text { a site is open })=0,
$$

which means that above some value of $\lambda$, Inequality (9) holds.

Inequality (10) is verified if:

$$
\frac{2(1+2 \beta \gamma) M}{\beta \gamma m} \leq \frac{(1-\varepsilon) \lambda \delta^{2}}{4},
$$

which can be recast as

$$
\gamma \geq \frac{8 M}{\beta\left[(1-\varepsilon) \delta^{2} \lambda m-16 M\right]} .
$$

When $\lambda \geq 16 M / \delta^{2} \varepsilon m$, a sufficient condition is

$$
\gamma \geq \frac{8 M}{\beta(1-2 \varepsilon) \delta^{2} \lambda m}:=\frac{c_{1}}{\lambda} .
$$

We thus proved that for sufficiently high densities, if $\gamma \geq$ $c_{1} / \lambda$, the origin belongs a.s. to a finite cluster in the discrete model. We now have to prove that in this case, the origin also belong to a finite cluster in the continuous model.

Because of Lemma 2, when a site is closed, the square centered on this site contains only isolated nodes (we will call it hereafter a closed square). Therefore, in the continuous model, when $\gamma \geq c_{1} / \lambda$, the origin is surrounded by a chain

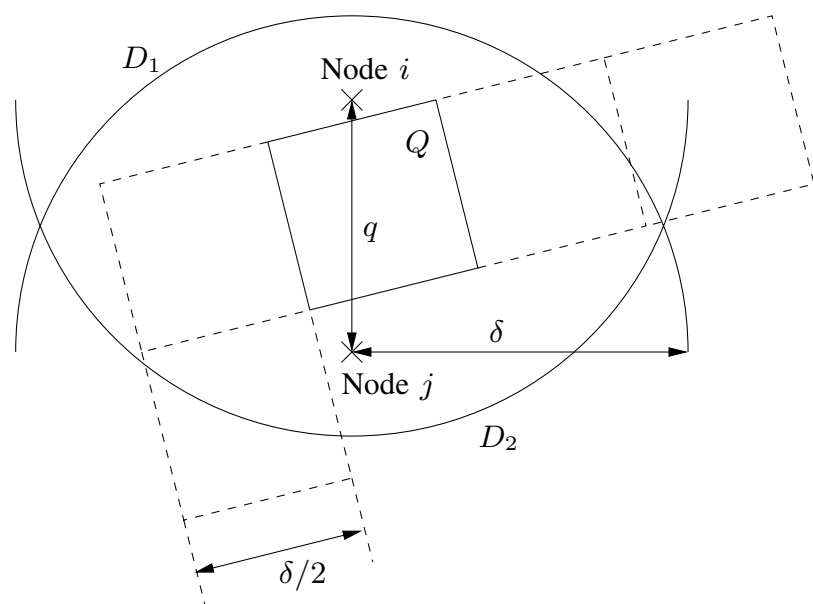

Fig. 4. The chain of closed squares separating the two nodes.

of closed squares with no link inside. To make sure that the origin belong to a finite cluster, we have to prove that no link can cross this chain.

Let us consider two nodes $i$ and $j$, such that Node $i$ is located inside a square centered on an open site connected to the origin (in the discrete model), and Node $j$ is located inside a square centered on an open site disconnected from the origin (i.e. on the other side of the chain). As between these nodes, there is a chain of closed squares, the distance between them $q:=\left\|x_{i}-x_{j}\right\|$ is larger than $\delta / 2$.

We consider two cases. First, we assume that $\delta / 2<q<\delta$. In this case we construct the disk $D_{1}$ of radius $\delta$ centered on $x_{i}$ and the disk $D_{2}$ of radius $\delta$ centered on $x_{j}$, as depicted in Figure 4. As the chain of closed squares separates $x_{i}$ and $x_{j}$, there exists at least one closed square $Q$ that has a non-empty intersection with the segment $\left[x_{i}, x_{j}\right]$. Moreover, the shortest distance between $\left[x_{i}, x_{j}\right]$ and $\mathbb{R}^{2} \backslash\left(D_{1} \cup D_{2}\right)$ is

$$
\sqrt{\delta^{2}-\frac{q^{2}}{4}} \geq \frac{\sqrt{3}}{2} \delta .
$$

As the diagonal of $Q$ has length $\delta / \sqrt{2}, Q$ cannot have a nonempty intersection with $\left[x_{i}, x_{j}\right]$ and with $\mathbb{R}^{2} \backslash\left(D_{1} \cup D_{2}\right)$ at the same time. Therefore $Q \subset D_{1} \cup D_{2}$.

Furthermore, we count the number of nodes inside three different subsets of $Q$ :

$$
\begin{aligned}
& N_{1}=\Phi_{\lambda}\left(Q \cap\left(D_{1} \backslash D_{2}\right)\right) \\
& N_{2}=\Phi_{\lambda}\left(Q \cap\left(D_{2} \backslash D_{1}\right)\right) \\
& N_{3}=\Phi_{\lambda}\left(Q \cap D_{1} \cap D_{2}\right) .
\end{aligned}
$$

As $Q$ is a closed square, we have by assumption $N_{1}+N_{2}+$ $N_{3} \geq 2 N^{\prime}$. This implies that either

$$
N_{1}+N_{3} \geq N^{\prime}
$$

or

$$
N_{2}+N_{3} \geq N^{\prime}
$$

Let us assume without loss of generality that the first inequality holds. There are thus at least $N^{\prime}$ nodes located inside $D_{1}$. As 
$D_{1}$ has radius $\delta$, the signal received by Node $i$ from each of these nodes is at least $\mathrm{Pm}$. The SINR at Node $i$ received from Node $j$ is thus upper-bounded by

$$
\beta_{j i} \leq \frac{P M}{N_{0}+\gamma N^{\prime} P m} .
$$

Plugging the value of $N^{\prime}$ into this expression, we obtain finally

$$
\beta_{j i} \leq \beta,
$$

and no link between Node $i$ and Node $j$ exists. The same is true if $N_{2}+N_{3} \geq N^{\prime}$.

Let us now address the case where $q>\delta$ (the case $q=\delta$ appears with probability zero). In this case, we draw the same disks $D_{1}$ and $D_{2}$, but with radius $q$. There exists at least one square $Q$ of the chain such that $Q \subset D_{1} \cup D_{2}$. We define $N_{1}$, $N_{2}$ and $N_{3}$ in the same way as above. Thus, either $N_{1}+N_{3} \geq$ $N^{\prime}$ or $N_{2}+N_{3} \geq N^{\prime}$.

Let us assume without loss of generality that $N_{1}+N_{3} \geq N^{\prime}$. This implies that there are at least $N^{\prime}$ nodes inside $D_{1}$. Node $j$ is by construction on the border of $D_{1}$. Therefore, all these nodes are closer to Node $i$ than Node $j$. As we assumed that $l(x)$ is decreasing, the SINR at $i$ from Node $j$ is bounded above by

$$
\beta_{j i} \leq \frac{P l(q)}{N_{0}+\gamma P N^{\prime} l(q)} .
$$

As $M>m$, from (8) we have

$$
N^{\prime}>\frac{1}{\beta \gamma}
$$

and thus

$$
\beta_{j i} \leq \beta,
$$

meaning that the link cannot exist.

Consequently, we have proved that if the discrete model is sub-critical, then the continuous model is sub-critical too. We conclude that when (12) holds, the network is sub-critical. This implies that the critical threshold is smaller than $c_{1} / \lambda$. This concludes the proof of Theorem 2 .

In particular, we have

$$
\lim _{\lambda \rightarrow \infty} \gamma^{*}(\lambda)=0,
$$

implying that for a fixed $\gamma$, the network eventually becomes disconnected when the node density increases. In this case, it would be interesting to decrease the required $\operatorname{SINR} \beta$ (which means decreasing the rate on the links) in order to reconnect the network. To finish this section, we will study the asymptotic behavior of the critical $\beta$ for percolation when $\gamma$ is fixed, that we will denote by $\beta^{*}(\lambda)$.

We observe in (11) that $\beta$ and $\gamma$ play the same role, as they are multiplied. Therefore, from this inequality, we can derive a condition on $\beta$ :

$$
\begin{aligned}
\beta & \geq \frac{8 M(1+2 \beta \gamma)}{(1-\varepsilon) \delta^{2} \gamma \lambda m} \\
& \geq \frac{8 M}{(1-\varepsilon) \delta^{2} \gamma \lambda m}
\end{aligned}
$$

The condition has exactly the same form as the condition on $\gamma$, and the same bound is valid for $\beta^{*}(\lambda)$ :

$$
\beta^{*}(\lambda) \leq \frac{c_{1}}{\lambda} .
$$

The two bounds (7) and (13) can be interpreted as follows: when the node density becomes large, because of the interferences, one must either use wider spectrum (i.e. decrease $\gamma$ ) or decrease the rate of the transmissions (i.e. decrease $\beta$ ). This establishes thus a trade-off between connectivity and capacity.

\section{B. A simple TDMA scheme}

In the previous section, we concluded that connectivity eventually becomes poor when the node density increases. This is however true only if all nodes emit at the same time. Actually, the poor connectivity is due to the interferences. If we set $\gamma=0$ in the model (we neglect interferences), connectivity improves with density. This suggests that with an appropriate strategy, good connectivity can be achieved in this case.

In this section, we propose to randomly divide the nodes into $t$ categories (or time slots), and to let only the nodes of one category emit at the same time. It has been shown in [2] that using this scheme with $t$ slots performs as well as having $\gamma=1 / t$.

In this section, we compute a lower bound on the necessary number of time slots $t^{*}(\lambda)$ to restore connectivity when $\gamma=1$ (no CDMA).

Theorem 3: If the attenuation function is decreasing and is such that (5) and (6) hold, there exists a constant $c_{2}>0$ such that

$$
t^{*}(\lambda)>\lambda c_{2} .
$$

The derivation of this lower bound is very similar to that of the previous section. We start thus with the same assumptions on $l(\cdot)$. As each node chooses its category randomly between 1 and $t$, the process formed by the nodes of a particular category is still a Poisson point process, but with intensity $\lambda / t$.

We divide the plane into squares in the same way as above, i.e. as depicted in Figure 3. We then construct a site percolation model on this grid by declaring a site of $\mathbb{Z}^{2}$ closed if the square centered on this site contain at least $2 N^{\prime}=2(1+2 \beta) M / \beta m$ nodes of each category. Thus, if a site is declared closed, during each time slot, according to Lemma 2 (with $\gamma=1$ ), the square is full of isolated nodes.

We compute now the probability that a site is closed. The number of nodes of each category in a square is a Poisson random variable of parameter $\delta^{2} \lambda / 2 t$. Let us call $N_{i}$ the variable counting the number of nodes of category $i$ in the square. We have

$$
\begin{aligned}
\mathbb{P}(\text { a site is closed }) & =\mathbb{P}\left(\bigcap_{i=1}^{t}\left\{N_{i}>2 N^{\prime}\right\}\right) \\
& =\prod_{i=1}^{t} \mathbb{P}\left(N_{i}>2 N^{\prime}\right) \\
& =\mathbb{P}^{t}\left(N_{i}>2 N^{\prime}\right)
\end{aligned}
$$


Therefore, if

$$
\mathbb{P}\left(N_{i}>2 N^{\prime}\right)>\sqrt[t]{1-p_{\text {site }}},
$$

where $p_{\text {site }} \cong 0.59$ is the site percolation threshold on $\mathbb{Z}^{2}$, we obtain

$$
\mathbb{P}(\text { a site is open })<p_{\text {site }} .
$$

For having (14), the intensity of the Poisson random variable $N_{i}$ has to be large enough. Let $\mu$ be the minimal intensity of a Poisson random variable $X_{\mu}$ such that $\mathbb{P}\left(X_{\mu}>2 N^{\prime}\right) \geq$ $\sqrt[t]{1-p_{\text {site }}}$. Then a sufficient condition for having (14) is

$$
\frac{\delta^{2} \lambda}{4 t}>\mu
$$

or equivalently

$$
t<\frac{\delta^{2} \lambda \mu}{4} .
$$

We obtained thus the bound on $t^{*}(\lambda)$ by proving that if

$$
t<\lambda c_{2}
$$

for some fixed constant $c_{2}=\delta^{2} \lambda \mu / 4$, the network is almost surely disconnected. This proves Theorem 3 .

As each category has a node density $\lambda / t$, and as $t>\lambda c_{2}$, we conclude that the node density of the nodes allowed to emit at each time slot is uniformly bounded above by $c_{2}$, independently from the number of nodes. This means that good connectivity can be achieved, but that nodes can emit less and less frequently. This result suggests that the rate throughput per node decreases like $1 / \lambda$.

However, our TDMA scheme is very simple, and our negative result is possibly due to its sub-optimality. In the next section, we study the transport capacity of dense networks with bounded attenuation function in a much more general context. We will see that the available rate per node has to decrease like $1 / \lambda$, no matter what scheduling or power allocation is used.

\section{Transport capacity}

In this section, we will prove that the total transport capacity of dense ad hoc networks is bounded above by a constant, independently from the power allocation strategy and number of nodes.

We consider a compact region $S$ of $\mathbb{R}^{2}$, in which the network is confined. We introduce furthermore the following technical assumption on the attenuation function:

$$
l(\|x-y\|)>0 \quad \forall x, y \in S .
$$

This condition is always verified if the attenuation is decreasing and has unbounded support.

Theorem 4: If the attenuation function is bounded and verifies (15), the total transport capacity of the network is bounded above by a constant, independently of the number of nodes.

As $S$ is a compact set, we have

$$
m:=\inf _{x, y \in S} l(\|x-y\|)>0 .
$$

Hereafter, we will denote by $P_{i}$ the actual power emitted by Node $i$, and by $P_{t o t}$ the total power emitted in the network. Hence

$$
P_{t o t}=\sum_{i} P_{i} .
$$

Let us pick an arbitrary real number $0<\varepsilon<1 / 2$, and call dominant the nodes $i$ such that $P_{i} / P_{t o t}>\varepsilon$. Clearly, there are no more than $1 / \varepsilon$ dominant nodes in the network.

Let us now look at the transport capacity. As above, we assume that Node $i$ is transmitting towards a destination $r(i)$ located at a distance $\delta_{i}$. The available SINR at Node $r(i)$ is

$$
\beta_{i, r(i)}=\frac{P_{i} l\left(\delta_{i}\right)}{N_{0}+\gamma I\left(x_{r(i)}\right)}
$$

with

$$
I\left(x_{r(i)}\right)=\sum_{k \neq i, r(i)} P_{k} l\left(\left\|x_{k}-x_{r(i)}\right\|\right) .
$$

As $l(\|x-y\|)>m$ inside $S$ by assumption, one can bound $I\left(x_{r(i)}\right)$ from below

$$
I\left(x_{r(i)}\right) \geq m\left(P_{t o t}-P_{i}-P_{r(i)}\right),
$$

and obtain a bound on $\beta_{i, r(i)}$

$$
\beta_{i, r(i)} \leq \frac{P_{i} l\left(\delta_{i}\right)}{N_{0}+\gamma m\left(P_{t o t}-P_{i}-P_{r(i)}\right)} .
$$

Then, let us consider two distinct classes of nodes. The first class $\mathcal{C}_{1}$ contains all nodes $i$ that are not dominant and emit towards a destination $r(i)$ that is not dominant. For these nodes, we use the following looser bound

$$
\beta_{i, r(i)} \leq \frac{P_{i} l\left(\delta_{i}\right)}{\gamma m P_{t o t}(1-2 \varepsilon)} .
$$

The second class $\mathcal{C}_{2}$ contains the nodes that do not belong to $\mathcal{C}_{1}$ (i.e. they are either dominant, or emit towards dominant node, or both). For these nodes, we use the following bound:

$$
\beta_{i, r(i)} \leq \frac{P_{\max } l\left(\delta_{i}\right)}{N_{0}} .
$$

We notice that the second class contains a finite number of nodes (this number is smaller than $2 / \varepsilon$ ).

We can now sum the individual transport capacities, and obtain the total capacity of the network. In a first step, we will add separately the transport capacities of the nodes of the first class:

$$
\begin{aligned}
\sum_{i \in \mathcal{C}_{1}} \delta_{i} C\left(\beta_{i, r(i)}\right) & \leq \sum_{i \in \mathcal{C}_{1}} \frac{1}{2} \delta_{i} \beta_{i, r(i)} \\
& \leq \sum_{i \in \mathcal{C}_{1}} \delta_{i} \frac{P_{i} l\left(\delta_{i}\right)}{2 \gamma m P_{t o t}(1-2 \varepsilon)} \\
& \leq \max _{i}\left[\delta_{i} l\left(\delta_{i}\right)\right] \sum_{i \in \mathcal{C}_{1}} \frac{P_{i}}{2 \gamma m P_{t o t}(1-2 \varepsilon)} \\
& \leq \max _{i}\left[\delta_{i} l\left(\delta_{i}\right)\right] \frac{P_{t o t}}{2 \gamma m P_{t o t}(1-2 \varepsilon)} \\
& =\frac{1}{2 \gamma m(1-2 \varepsilon)} \max _{i}\left[\delta_{i} l\left(\delta_{i}\right)\right] .
\end{aligned}
$$


We next add up the transport capacities of the nodes of the second class:

$$
\begin{aligned}
\sum_{i \in \mathcal{C}_{2}} \delta_{i} C\left(\beta_{i, r(i)}\right) & \leq \sum_{i \in \mathcal{C}_{2}} \frac{1}{2} \delta_{i} \beta_{i, r(i)} \\
& \leq \sum_{i \in \mathcal{C}_{2}} \frac{P_{\max }}{2 N_{0}} \delta_{i} l\left(\delta_{i}\right) \\
& \leq \frac{P_{\max }}{\varepsilon N_{0}} \max _{i}\left[\delta_{i} l\left(\delta_{i}\right)\right] .
\end{aligned}
$$

The total transport capacity is thus bounded above by

$$
C_{\text {tot }} \leq\left(\frac{P_{\max }}{\varepsilon N_{0}}+\frac{1}{2 \gamma m(1-2 \varepsilon)}\right) \max _{i}\left[\delta_{i} l\left(\delta_{i}\right)\right] .
$$

As $l(\cdot)$ is uniformly bounded and decreases at least as fast as $x^{-2}$, the function $x l(x)$ is also bounded. This ensures that the above maximum exists and is uniformly bounded, even if the number of nodes goes to infinity.

We have therefore proved that the total transport capacity of the network is uniformly bounded. Assuming a uniform traffic matrix for example, this implies that the available bandwidth per node decreases like $1 / n$.

\section{CONCLUSION}

In this paper, we showed the strong impact of the shape of the attenuation function on the properties of dense ad hoc networks. The commonly used power law attenuation confers to the model particular scaling properties. We used the STIRG model defined in [2] to explain that in this case, connectivity improves when the node density increases. The transport capacity under these specific assumptions has been proven to grow like the square root of the number of nodes, by Gupta and Kumar [1].

We showed however that these results rely on the specific shape of the power law function. In fact, if one uses a bounded attenuation function, that does not diverge at the origin, both connectivity and capacity results are much more pessimistic. We proved that the critical value of the orthogonality coefficient $\gamma^{*}(\lambda)$ must decrease at least as fast as $1 / \lambda$ when $\lambda \rightarrow \infty$, which means that for a fixed value $\gamma$, the network will become almost surely made of finite, disconnected clusters. To keep the network well connected, one has to reduce the minimal SINR required threshold $\beta^{*}(\lambda)$ also like $1 / \lambda$, or to limit the number of nodes that emit at the same time, independently of the total number of nodes in the network. In both cases, connectivity is maintained, but at the expense of a decreased capacity. There is therefore a fundamental trade-off between connectivity and capacity. We also proved that the total transport capacity remains constant when the number of nodes increases, provided that the attenuation function has a support larger than the diameter of the network. This latter result leads to much lower available rates per node (of the order of $1 / n$ ) than in the Gupta and Kumar case (which was of the order of $1 / \sqrt{n}$ ).

Physics predicts that the attenuation function has to be bounded at the origin. Empirical models for attenuation functions are usually piecewise power laws, valid for large scale area, but with a smaller fading exponent close to the radiating antenna. Other models (Rician, etc) need to be applied close to the origin [4]. One suspects that the fading exponent tends to zero when distances are very small. It remains to evaluate by how much, in practice, ad hoc or sensor networks will have a density large enough to invalidate a pure power law fading. It is however clear that fundamental results on the connectivity and capacity of dense ad hoc networks, which are almost the only ones we have to date, strongly depend on the behavior of the attenuation function at small scales.

\section{APPENDIX}

We consider a function that fulfill the following property:

$$
\frac{l(a x)}{l(a y)}=\frac{l(x)}{l(y)} \quad \forall x, y, a>0
$$

It follows from the above equality that

$$
\frac{l(a x)}{l(x)}=\frac{l(a y)}{l(y)}=c(a),
$$

because $x$ and $y$ can be arbitrary chosen. Thus we have

$$
l(a x)=c(a) l(x), \quad \forall a, x \neq 0 .
$$

By letting $x=1$ in this last equality, we find that

$$
c(a)=\frac{l(a)}{l(1)}
$$

and thus

$$
l(a x)=\frac{l(a) l(x)}{l(1)} \quad \forall a, x \neq 0
$$

Let us consider now a transform of $l$ :

$$
L(x):=\log \left(l\left(e^{x}\right)\right)
$$

This new function is affine:

$$
\begin{aligned}
L(x+y) & =\log \left[l\left(e^{x+y}\right)\right] \\
& =\log \left[l\left(e^{x} e^{y}\right)\right] \\
& =\log \left[\frac{l\left(e^{x}\right) l\left(e^{y}\right)}{l(1)}\right] \\
& =\log \left[l\left(e^{x}\right)\right]+\log \left[l\left(e^{y}\right)\right]-\log [l(1)] \\
& =L(x)+L(y)-L(0),
\end{aligned}
$$

and thus

$$
L(x)=\alpha x+L(0)
$$

for some constant $\alpha$. We finally compute then the original function $l(x)$ :

$$
\begin{aligned}
l(x) & =\exp \{L[\log (x)]\} \\
& =\exp [\alpha \log (x)+L(0)]=l(1) x^{\alpha} .
\end{aligned}
$$

\section{ACKNOWLEDGMENT}

We would like to thank Prof. François Baccelli, ENSINRIA, for fruitful discussions during the preparation of this paper. 


\section{REFERENCES}

[1] P. Gupta and P. R. Kumar, "The capacity of wireless networks," IEEE Trans. Inform. Theory, vol. 46(2), pp. 388-404, Mar. 2000.

[2] O. Dousse, F. Baccelli, and P. Thiran, "Impact of interferences on connectivity of ad hoc networks," in Proc. IEEE Infocom, San Francisco, Apr. 2003.

[3] G. R. Grimmett, Percolation. Springer, 1999.

[4] H. Hashemi, "The indoor radio propagation channel," Proceedings of the IEEE, vol. 81(7), pp. 943-968, July 1993.

[5] R. J. C. Bultitude and D. A. Hughes, "Propagation loss at $1.8 \mathrm{ghz}$ on microcellular mobile radio channels," in Proc. PIMRC'96, Oct. 1996.

[6] E. N. Gilbert, "Random plane networks," SIAM J., vol. 9, pp. 533-543, 1961.

[7] R. Meester and R. Roy, Continuum percolation. Cambridge University Press, 1996.

[8] T. K. Philips, S. S. Panwar, and A. N. Tantawi, "Connectivity properties of a packet radio network model," IEEE Trans. Inform. Theory, vol. 35, pp. 1044-1047, Sept. 1989.

[9] S. Quintanilla, S. Torquato, and R. M. Ziff, "Efficient measurement of the percolation threshold for fully penetrable discs," J. Phys. A: Math. Gen., vol. 33, pp. L399-L407, Oct. 2000.

[10] L. Booth, J. Bruck, M. Franceschetti, and R. Meester, "Continuum percolation and the geometry of wireless networks," Annals of Applied Probability, vol. 13(2), pp. 722-731, 2003.

[11] O. Dousse, P. Thiran, and M. Hasler, "Connectivity in ad-hoc and hybrid networks," in Proc. IEEE Infocom, New York, June 2002.
[12] L. Booth, J. Bruck, M. Cook, and M. Franceschetti, "Ad hoc wireless networks with noisy links," in Proc. ISIT, Yokohama, Japan, 2003.

[13] F. Baccelli and B. Blaszczyszyn, "On a coverage process ranging from the boolean model to the poisson voronoi tessellation, with applications to wireless communications," Adv. Appl. Prob., vol. 33(2), pp. 293-323, 2001.

[14] P. Gupta and P. R. Kumar, "Critical power for asymptotic connectivity in wireless networks," Stochastic Analysis, Control, Optimization and Applications: A Volume in Honor of W.H. Fleming, 1998, edited by W.M. McEneany, G. Yin, and Q. Zhang, (Eds.) Birkhäuser.

[15] F. Xue and P. R. Kumar, "The number of neighbors needed for connectivity of wireless networks," Wireless Networks, to appear.

[16] S. Shakkottai, R. Srikant, and N. Shroff, "Unreliable sensor grids: Coverage, connectivity and diameter," in Proc. IEEE Infocom, San Francisco, Apr. 2003.

[17] L.-L. Xie and P. R. Kumar, "A network information theory for wireless communication: Scaling laws and optimal operation," IEEE Trans. Inform. Theory, submitted for publication.

[18] O. Lévêque, E. Telatar, and D. Tse, "Upper bounds on the capacity of ad-hoc wireless networks," in Proc. Winter School on Coding and Information Theory, Monte Verità, Switzerland, Feb. 2003.

[19] M. Gastpar and M. Vetterli, "On the capacity of wireless networks: the relay case," in Proc. IEEE Infocom, New York, June 2002.

[20] M. Grossglauser and D. Tse, "Mobility increases the capacity of ad-hoc wireless networks," in Proc. IEEE Infocom, Anchorage, Alaska, Apr. 2001.

[21] B. Liu, Z. Liu, and D. Towsley, "On the capacity of hybrid wireless networks," in Proc. IEEE Infocom, San Francisco, Apr. 2003. 